\title{
Periodic solutions of non linear differential difference equations*
}

By Stephane Laederich, IMA, University of Michigan, Ann Arbor, MI, USA

\section{Introduction}

In this paper, we will be mainly looking at a chain of gravitational pendula coupled by torsion springs and forced by some mechanical device (see fig. 1). We are interested in the behaviour of the system near its stable equilibrium, that is when each of the pendula is near its lower equilibrium.

The equation describing such systems is of the form

$$
\begin{aligned}
& -v_{1} \phi_{1}+v_{2} \phi_{2}=L_{1}\left(\phi_{1}\right)+f_{1}(t)+h_{1}\left(t, \phi_{1}, \frac{d}{d t} \phi_{1}\right), \\
& v_{k+1} \phi_{k+1}-2 v_{k} \phi_{k}+v_{k+1} \phi_{k+1} \\
& \quad=L_{k}\left(\phi_{k}\right)+f_{k}(t)+h_{k}\left(t, \phi_{k}, \frac{d}{d t} \phi_{k}\right), \quad k=2, \ldots, N-1, \\
& -v_{N} \phi_{N}+v_{N-1} \phi_{N-1}=L_{N}\left(\phi_{N}\right)+f_{N}(t)+h_{N}\left(t, \phi_{N}, \frac{d}{d t} \phi_{N}\right),
\end{aligned}
$$

where $L_{k}$ is the second order differential operator

$$
L_{k}=\frac{d^{2}}{d t^{2}}+\gamma_{k} \frac{d}{d t}+\mu_{k}
$$

The $f_{k}$ 's and $h_{k}$ 's will be real or complex valued functions which are 1 -periodic in $t$. The $\phi$ 's are the angles between the vertical and the pendula, the $\mu$ 's are given by the gravitational constant divided by the length of the pendula, the $\gamma$ 's are the friction terms and the $v$ 's are the coupling constants. Note that the first and last equation in (1.1) correspond to boundary conditions. These chains of coupled pendula arise in various settings. They can be used, for example to model coupled Josephson Junctions as de-

* Research partially supported by AFOSR under U.R.I. contract F49620-86-C-0131 to Northeastern University. 
Figure 1
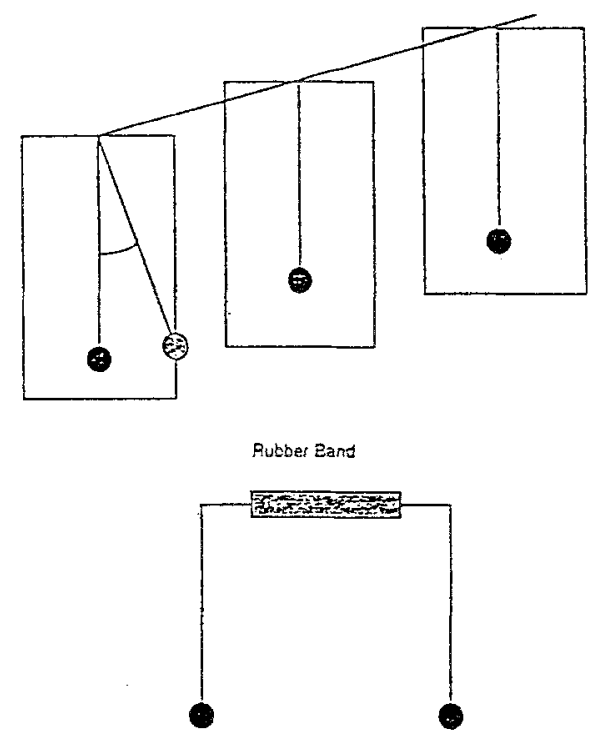

scribed by M. Levi [Le]. Equation (1.1) would then represent the linearisation around the lower equilibrium. We will consider long chains, that is, large $N$. We will assume that there are $C^{(2)}$ functions $v(x), \mu(x), \gamma(x)$, $f(t, x)$ and $h(t, x, u, v)$ with the following properties:

$$
\begin{aligned}
& \gamma_{k}=\gamma\left(\frac{k}{N}\right), \quad v_{k}=v\left(\frac{k}{N}\right), \quad \mu_{k}=\mu\left(\frac{k}{N}\right), \\
& f_{k}(t)=\frac{1}{N} f\left(t, \frac{k}{N}\right), \quad h_{k}\left(t, \frac{u}{N}, \frac{v}{N}\right)=\frac{1}{N^{2}} h\left(t, \frac{k}{N}, u, v\right)
\end{aligned}
$$

These assumptions are just smoothness assumptions in the variable $k$ for the coefficients of (1.1). The forcing term is scaled so as to allow solutions to be $O(1 / N)$ and the nonlinearity is scaled because it is quadratic in the $\phi$ 's. The scaling (1.3) can also be motivated by thermodynamical limits: Keeping the length of the chain constant, one increases the number $N$ of "identical" pendula. The natural limit, which in this case is a singularly perturbed partial differential equation is described in [La]. The striking fact is that equation (1.1) under the assumptions given by (1.3) behaves as if it was a linear uncoupled system. That is, for large $N$, neither the left hand side nor the non linearity do matter in (1.1). In fact, under the correspondence

$$
\phi_{k}(t)=\frac{1}{N} u\left(t, \frac{k}{N}\right)
$$


solutions $u$ of

$$
\left(\frac{d^{2}}{d t^{2}}+\gamma(x) \frac{d}{d t}+\mu(x)\right) u(t, x)+f(t, x)=0,
$$

are a good approximation of solutions of (1.1). This equation is the zeroth order equation of the partial differential equation arising in the thermodynamical limit of (1.1).

Equation (1.1) is just a particular case of a more general class of differential difference equations. We will consider problems of the form:

$$
A \phi=L(\phi)+f(t)+h\left(t, \phi, \frac{d}{d t} \phi, \ldots, \frac{d^{k}}{d t^{k}} \phi\right), \quad \phi \in C^{N},
$$

where $A$ is a $N \times N$ matrix with constant coefficients, $L$ is a matrix differential operator in $t$ with constant coefficients, $f$ and $h$ are $C^{N}$ valued functions and $t \in S^{1}$. We will assume that $h(t, 0)=0$. A typical example has $A$ equal the tridiagonal matrix arising from the discretization of the Laplacian, for example in the discretization of a rod equation. Another example of systems leading to equations of the type of (1.3) are free planar chains. Looking for periodic solutions for a free chain (see [LL]) leads to a system of equations of the form of (1.5). Several other systems lead to equations of this form. For example, a model of fish swimming, (see G. Bowtell [B]) or the model for the travelling neural waves in a fish (see G. B. Ermentrout and N. Kopell [EK]).

We will first prove existence and uniqueness of periodic solutions for such systems. This can be done in several ways, for example as in J. Hale $[\mathrm{H}]$ and J. Hale and G. Raugel [HR] using the dissipative nature of the equations. We prefer to use a more direct approach, also based on a contraction argument which allows us to obtain more information about the nature of the periodic solution. Verifying the assumptions of this theorem for the particular case of (1.1) will prove the existence of a periodic solution of (1.1). Using the methods of the proof and a simple Taylor series argument will allow us to show that (1.1) behaves as if there was no coupling.

\section{Results}

We can now state our results. Since we are working with time periodic functions, we will work on $\left[H^{l}\left(S^{1}\right)\right]^{N}$. More specifically, for

$$
\phi=\left(\begin{array}{c}
\phi \\
\vdots \\
\phi_{N}
\end{array}\right) \in\left[H^{l}\left(S^{1}\right)\right]^{N},
$$


let

$$
\|\phi\|_{l}=\left[\sum_{k=1}^{N}\left\|\phi_{k}\right\|_{H^{l}\left(S^{1}\right)}^{2}\right]^{1 / 2} .
$$

We will denote the completion of $\left[H^{l}\left(S^{1}\right)\right]^{N}$ with respect to \|\| by $\mathscr{H}_{l}$. Since $L$ is a matrix differential operator in $t$ with constant coefficients, applying $L$ to the vector $1 e^{\text {int }}$,

$$
\begin{aligned}
& \boldsymbol{I}=\left(\begin{array}{c}
1 \\
\vdots \\
1
\end{array}\right) \text {, we can naturally define a } N \times N \text { matrix } L(n) \text { as follows: } \\
& L\left(1 e^{\mathrm{int}}\right)=L(n) 1 e^{\mathrm{int}}
\end{aligned}
$$

In order to solve the non homogeneous linear equation, we will require that for all $n$ 's,

$$
A-L(n) \text { is invertible, }
$$

there are constants $\Lambda_{0}, \Lambda_{1} \in \boldsymbol{R}_{+}, p_{0}, p_{1} \in N$ such that

$$
\Lambda_{0} n^{p_{0}} \leq\|A-L(n)\| \leq \Lambda_{1} n^{p_{1}}, \quad \forall n \in N .
$$

Here \|\| denotes the natural sup norm on $C^{N}$ for matrices. This condition on the matrix $A$ and on the operator $L$ enables us to avoid the appearance of resonances and will be needed for the contraction argument. In the case of eq. (1.1), it will be shown that these conditions are satisfied only when the $\gamma_{k}$ 's are non zero, i.e. the pendula are damped. For simplicity of notation, let $\mathscr{B}_{k}(R)$ be the ball of radius $R$ in $C^{(k)}$. For a $C^{(l)}$ function $h\left(t, x_{1}, \ldots, x_{k}\right)$ with values in $C^{N}$ let

$$
\|h\|_{R, \beta, j, i}=\sup _{\substack{t \in S^{1} \\ x \in \mathscr{B}_{k}(R)}}\left|D_{i}^{i} D_{x}^{\beta} h_{j}\left(t, x_{1}, \ldots, x_{k}\right)\right|,
$$

where $\beta \in N^{k}, i \in N$ and $j=1, \ldots, N$. We will denote the maximum of the $\|h\| \|_{R, \beta, j, i}$ for $|\beta| \leq l+1, i \leq l$ over all $j=1, \ldots, N$ by $\|h\|_{R}$. We can now state:

Theorem 2.1. Let $A, L$ be as above and assume (2.3) holds. Let $h\left(t, x_{1}, \ldots, x_{k}\right)$ be $C^{(i)}$ in $t$ and $C^{(l+1)}$ in each of the $x_{m}$. Assume that $k \leq p_{0}$. Let $f(t) \in \mathscr{H}_{l}, l \geq \operatorname{deg} L+1-p_{0}+k$. Then there are constants $R$ and $K$ such that if
(i) $\|h\|_{R} \leq K$
(ii) $h(t, 0, \ldots, 0)=0$, 
then

$$
A \phi=L(\phi)+f(t)+h\left(t, \phi, \frac{d}{d t} \phi, \ldots, \frac{d^{k}}{d t^{k}} \phi\right),
$$

has a unique classical solution $\phi \in \mathscr{H}_{l}$, satisfying $\sup _{t \in S^{1}}\|\phi(t)\| \leq R$.

Furthermore

$$
R=0\left(1+\frac{1}{\Lambda_{0}}\|f\|_{l}\right)
$$

and

$$
K=0\left(\Lambda_{0} \cdot\left(\|f\|_{l}+1\right)^{-1}\right) .
$$

Here \|\| denotes the natural $C^{(N)}$ norm. We will keep this notation throughout the paper. Note that $h$ can at most depend on derivatives of $\phi$ of order lower or equal to the order of $L$. Furthermore, when $h$ depends only on $\phi$, the forcing function $f$ needs only to lie in $\mathscr{H}_{l}$ where $l \geq \operatorname{deg} L+1-p_{0}$. We will also prove

Corollary 2.2. The conclusions of the previous theorem hold true for (1.1) provided there exist constants $\underline{v}, \bar{v}, \underline{\mu}, \bar{\mu}, b$ and $c$ such that

(i) $0 \leq \underline{v} \leq v_{k} \leq \bar{v}, \mu_{k} \leq \bar{\mu}, \gamma \leq \gamma_{k} \leq \bar{\gamma}, k=1, \ldots, N$,

(ii) $\max _{k=2, \ldots, N}\left|\gamma_{k}-\gamma_{k-1}\right| \leq b / N, \max _{k=1, \ldots, N}\left|v_{k}-v_{k-1}\right| \leq c / N$,

(iii) $\bar{v}^{-2} \underline{\gamma} \geq \frac{2}{N} \frac{b \bar{v}+c \bar{\gamma}}{\underline{v}^{2}}$.

These conditions are needed to verify that in this case (2.3) holds. Note that if, for example, $v_{k}=1, k=1, \ldots, N, A$ has a zero eigenvalue. In view of the correspondence given by (1.3), the conditions of Corollary 2.2 can be interpreted as conditions on $\mu, \gamma, v$, their derivatives and $N$ : (i) represents $C^{0}$ bounds on $\mu, \gamma$ and $v$, (ii) requires $C^{(1)}$ bounds on $v$ and $\gamma$ and finally (iii) requires $N$ to be sufficiently large.

Theorem 2.1 will be proved by a contraction argument. We will construct an operator on $\mathscr{H}_{l}$ whose fixed point is the desired solution.

Assuming that the conditions of Corollary 2.2 are satisfied, that the linear terms in the $h_{k}$ 's are of order $1 / N$ and that the functions $\mu, v, \gamma, f$ and $h$ defined by (1.3) are smooth enough, we have:

Theorem 2.3. Let $\phi(t)$ be a solution of (1.1). Assuming that $f, \mu, v$ are $C^{(2)}$ in $x$, let

$$
u_{0}(t, x)=-\sum \frac{f^{(n)}(x)}{\mu(x)-n^{2}+i \gamma(x) n} e^{\mathrm{int}},
$$


where the $f^{(n)}$ are the Fourier coefficients of $f(t, x)$. Then there exists a constant $K_{1}$, independent of $N$ such that

$$
\sup _{t \in S^{1}}\left|\phi_{k}(t)-\frac{1}{N} u_{0}\left(t, \frac{k}{N}\right)\right| \leq \frac{K_{1}}{N^{(3 / 2)}},
$$

as $N \rightarrow \infty$. Furthermore, $K_{1}=0\left(\left(\sup _{x}\|f\|_{H^{3}\left(S^{1}\right)}\right)^{2}\right)$.

Equation (1.4) can be considered as the zeroth order equation for a singularly perturbed wave equation (see [La]). In this case $u_{0}$ is the zeroth order solution of the wave equation and one has a direct relation between (1.1) and a partial differential equation. This theorem has the practical advantage of providing information on (1.1) without having to invert large $N \times N$ matrices. The only tools required to prove this theorem are Taylor series.

Note that the use of Sobolev spaces may seem artificial for a system of ordinary differential equations. In fact the difference equation structure leads us naturally to such spaces. Furthermore, we get better differentiability results than the natural ones for ODE's. Namely, in (2.3) (ii), the worst possible case is $p_{0}=0$. In this case the solution is as smooth as $f$. As soon as $p_{0} \geq 1$, one starts gaining derivatives. Note also that the conditions on the nonlinearity are not optimal. One could require less differentiability.

In the next section, we will prove Theorem 2.1 and its corollary. Theorem 2.3 will be proved in the last section. This paper was written in partial fulfillment of the requirement of the Ph.D degree under direction of Nancy Kopell. I would like to thank Nancy Kopell for the many fruitful discussions we had and also would like to thank A. Lesniewski for his useful suggestion in the proof of Lemma 3.7.

\section{Existence proofs}

\subsection{Proof of Theorem 2.1}

For simplicity, we will let

$$
A_{n}=A-L(n), \quad n \in N \text {. }
$$

When $f(t)=\bar{f} e^{\mathrm{int}}, \bar{f} \in C^{N}$, the linear nonhomogeneous part of (1.3) has the simple solution

$$
\phi(t)=\left(A_{n}^{-1} \bar{f}\right) e^{\text {int }} .
$$

To see this, we make the Ansatz

$$
\phi(t)=\bar{\phi} e^{\mathrm{int}},
$$


where $\bar{\phi}$ is a constant vector of $\boldsymbol{C}^{N}$. The linear non homogeneous part of (1.3) can then be reduced to the time independent equation

$$
A \phi=L(n) \bar{\phi}+\bar{f},
$$

which has the desired solution. We now generalize this to any $f(t) \in \mathscr{H}_{l}$.

Definition 3.1. Let $A, L$ be as in Theorem 2.1 and let $f(t) \in \mathscr{H}_{l}, l \geq p_{1}$. For $\phi \in \mathscr{H}_{l}$ define

$$
\begin{aligned}
& B \phi=\sum_{n} A_{n} \phi^{(n)} e^{\mathrm{int},} \\
& \Omega(\phi)=B \phi-f(t),
\end{aligned}
$$

where

$$
\phi(t)=\sum_{n} \phi^{(n)} e^{\mathrm{int}}, \quad \phi^{(n)} \in C^{N}
$$

and where

$$
f(t)=\sum_{n} f^{(n)} e^{\mathrm{int}}, f^{(n)} \in C^{N} .
$$

It is easy to check that a solution $\phi$ of the linear non homogeneous part of (1.3) satisfies

$$
\Omega(\phi)=0 .
$$

To prove this assertion, expand $\phi(t)$ in Fourier series. The linear non homogeneous part of (1.5) is formally solved when for each $n \in N$,

$$
A \phi^{(n)}=L(n) \phi^{(n)}+f^{(n)}
$$

Now

$$
\Omega(\phi)=\sum_{n} A_{n} \phi_{(n)} e^{\mathrm{int}}-\sum_{n} f^{(n)} e^{\mathrm{int}} .
$$

Since $A_{n}=a-L(n)$, when $\phi$ is a solution to the linear non homogeneous part of $(1.5), \Omega(\phi)=0$. Note that the definitions of $\Omega, B$ are formal. Again, formally, we can compute the inverses of $\Omega$ and $B$.

Definition 3.2. Let $A, L$ be as in Theorem 2.1, let $f(t) \in \mathscr{H}_{l}, l \geq 0$. For $\phi \in \mathscr{H}_{l}$ define

$$
\begin{aligned}
& B^{-1} \phi=\sum A_{n}^{-1} \phi^{(n)} e^{\mathrm{int}}, \\
& \Omega^{-1}(\phi)=B^{-1}(\phi+f(t)) .
\end{aligned}
$$


The following proposition shows that the operators $B, \Omega$ and their inverses are well defined.

Proposition 3.3. Under the assumption (2.3), the following holds for any $f \in \mathscr{H}_{l}$ : For $l \geq p_{1}$,

(i) $\Omega, B: \mathscr{H}_{l} \rightarrow \mathscr{H}_{l-p_{1}}$,

and for $l \geq 0$,

(ii) $\Omega^{-1}, B^{-1} ; \mathscr{H}_{l} \rightarrow \mathscr{H}_{l+p_{0}}$,

where $p_{0}, p_{1}$ are as in (2.3).

Proof. The proof follows from straightforward estimates on the $l$-norms of $\Omega(\phi), B \phi$, and their inverses. We will prove the proposition for $B$. Denote $d / d t$ by $\nabla$ and let $m \leq l-p_{1}, M$ be fixed integers. Then, for

$$
\phi(t)=\sum_{h} \phi^{(n)} e^{\mathrm{int}} \in \mathscr{H}_{l},
$$

we have

$$
\nabla^{m} \sum_{|n| \leq M} A_{n} \phi^{(n)} e^{\text {int }}\left\|_{0}^{2}=\right\| \sum_{|n| \leq M}(\text { in })^{m} A_{n} \phi^{(n)} e^{\text {int }} \|_{0}^{2} .
$$

By the definition of \|\|$_{l}$, the right hand side is equal to

$$
\sum_{k=1}^{N} \sum_{|n| \leq M} n^{2 m}\left|\left[A_{n} \phi^{(n)}\right]_{k}\right|^{2}
$$

Since $m$ is finite, using (2.3) we can estimate this by

$$
\Lambda_{1}^{2} \sum_{|n| \leq M} n^{2 m+2 p_{1}}\left\|\phi^{(n)}\right\|^{2}
$$

but since $\phi \in \mathscr{H}_{l}$, we can estimate (3.3) from above by

$$
\Lambda_{\mathrm{L}}^{2}\|\phi\|_{l}^{2}
$$

We let $M$ tend to $\infty$ to infer

$$
\left\|\nabla^{m} B \phi\right\|_{0}^{2} \leq \Lambda_{1}^{2}\|\phi\|_{l}^{2} \text {. }
$$

By the definition of \|\|$_{l},\|B \phi\|_{l-p_{1}}^{2}=\sum_{m=0}^{l-p_{1}}\left\|\nabla^{m} B \phi\right\|_{0}^{2}$. We therefore have

$$
\|B \phi\|_{l-p_{1}} \leq(1+l) \Lambda_{1}\|\phi\|_{l} .
$$

Similarly, we obtain

$$
\left\|B^{-1} \phi\right\|_{l+p_{0}} \leq \frac{(1+l)}{\Lambda_{0}}\|\phi\|_{l} .
$$


By (3.3) and Proposition 3.3, we can solve the linear non homogeneous part of (1.3). For $R>0$ and $l \geq k+1$ define $\|h\|_{R}$ as in (2.4). Let $C$ be the Sobolev constant such that for $\phi \in \mathscr{H}_{l}$

$$
\sup _{t \in S^{1}}\left\{\|\phi\|, \ldots,\left\|\frac{d^{k}}{d t^{k}} \phi\right\|\right\} \leq C\|\phi\|_{l} \text {. }
$$

Since $l \geq k+1$, the existence of such a constant follows easily from the Sobolev inequalities. (See Pazy [Pa]). Let

$$
M(h)=|\|h\||_{C^{-1} R} \text {. }
$$

For the simplicity of notation, we will write

$$
H(\phi)=h\left(t, \phi, \ldots, \frac{d^{k}}{d t^{k}} \phi\right) .
$$

We have the simple

Lemma 3.4. Let $H(t, \phi)$ be as above. Then for any $l \geq k+1$, the mapping $H: \phi \rightarrow H(\phi)$ takes $\mathscr{H}_{l}$ into $\mathscr{H}_{l-k}$. Furthermore, if $\phi, \psi \in \mathscr{H}_{l}$, $\|\phi\|_{l},\|\psi\|_{l} \leq R$, we have

$$
\begin{aligned}
& \|H(t, \phi)\|_{l-k} \leq q_{1}\left(\|\phi\|_{l}\right), \\
& \|H(t, \phi)-H(t, \psi)\|_{l-k} \leq q_{2}(2 R)\|\phi-\psi\|_{l},
\end{aligned}
$$

where $q_{1}, q_{2}$ are polynomials of degree $l-k$ in their argument whose coefficients are linear in $M(h)$.

For a proof of this lemma, we refer to Palais [P] or to [La]. With this preliminary work done, we can define an operator on $\mathscr{H}_{l}$ whose fixed point will be the desired solution of (1.3).

Definition 3.5. Let $A, L$ be as in Theorem 2.1, let $f \in \mathscr{H}_{l}, l \geq \operatorname{deg} L+$ $1-p_{0}+k$. For any $h$, which is $C^{(1)}$ in $t, C^{(l+1)}$ in its other $k$ variables, define for $\phi \in \mathscr{H}_{l}$,

$$
T(\phi)=\Omega^{-1}(H(t, \phi)),
$$

where $H(t, \phi)$ is defined by (3.7). By Lemma $3.4, T$ is well defined on $\mathscr{H}_{l}$ and maps $\mathscr{H}_{l}$ into $\mathscr{H}_{l+p_{0}-k}$. Assume $H$ to be such that

$$
H(t, 0)=0 \quad \forall t \in S^{1} .
$$

Assume now that $\phi$ is a fixed point of $T$. This implies that

$$
\Omega(\phi)=H(t, \phi) \text {. }
$$

But by construction $\Omega: \phi \rightarrow A \phi-L(\phi)-f(t)$. Hence, if $\Omega(\phi)=H(t, \phi), \phi$ is a solution of (1.3). We now show that $T$ is a contraction. 
Proposition 3.6. Let $A, L$ be as in Theorem 2.1, let $l \geq \operatorname{deg} L+1-$ $p_{0}+k$ and let $f \in \mathscr{H}_{l}$. Denote the ball of radius $R$ in $\mathscr{H}_{l}$ by $U(R)$. Then there are constants $R, \chi$ such that if $M(h) \leq \chi$ then

$T: U(R) \rightarrow U(R)$,

and is a contraction.

Since $l \geq \operatorname{deg} L+1-p_{0}+k$ and since $T: \mathscr{H}_{l} \rightarrow \mathscr{H}_{l+p_{0}-k}$, a fixed point of $T$ will lie in $\mathscr{H}_{L+1}$. Hence, by Sobolev's embedding theorems, see [Pa], $\phi$ will be a classical solution. Thus, the proposition will conclude the proof of Theorem 2.1.

Proof. Using Lemma 3.4 and Proposition 3.3, if $\phi \in U(R)$,

$$
\begin{aligned}
\|T(\phi)\|_{l+p_{0}-k} & =\left\|B^{-1}(h(t, \phi)+f(t))\right\|_{l} \\
& \leq\left\|B^{-1}\right\|\left(q_{1}(R)+\|f\|_{l}\right),
\end{aligned}
$$

so by (3.5) we have

$$
\|T(\phi)\|_{l+p_{0}-k} \leq \frac{1+l}{\Lambda_{0}}\left(q_{1}(R)+\|f\|_{l}\right) .
$$

Similarly, by Lemma 3.4 , for $\phi, \psi \in U(R)$

$$
\|T(\phi)-T(\psi)\|_{l+p_{0}-k} \leq \frac{1+l}{\Lambda_{0}} q_{2}(2 R)\|\phi-\psi\|_{l} .
$$

Choose $R \geq\left((1+l) / \Lambda_{0}\right)\left(\|f\|_{l}\right)+1$ and choose $\chi$ such that

$$
\frac{1+l}{\Lambda_{0}} q_{1}(R), \quad \frac{1+l}{\Lambda_{0}} q_{2}(2 R)<1 \quad \text { if } M(h) \leq \chi .
$$

This implies that $T$ is a contraction provided $M(h)<\chi$. Note that when $h$ contains linear terms in $\phi$, the smallness of $M(h)$ implies that the coefficients of the linear terms are dominated by the ones coming from $L(\phi)$.

\subsection{Proof of Corollary 2.2}

Since we will be mostly working with tridiagonal matrices, let us introduce the following notation. For $U, W \in C^{N-1}, V \in C^{N}$,

$$
\begin{aligned}
& U=\left(u_{1}, \ldots, u_{N-1}\right), \\
& W=\left(w_{1}, \ldots, w_{N-1}\right), \\
& V=\left(v_{1}, \ldots, v_{N}\right),
\end{aligned}
$$


let

$$
J(U, V, W)=\left(\begin{array}{lll}
v_{1} & w_{1} & \\
u_{1} & & 0 \\
& & w_{N-1} \\
0 & & \\
& u_{N-1} & v_{N}
\end{array}\right]
$$

We need only to show that the conditions (i) $\rightarrow$ (iii) of the corollary imply (2.3). For technical reasons, we will change notation in (1.1): Let $\psi_{k}=v_{k} \phi_{k}$. The $\psi_{k}$ 's satisfy

$$
A \psi=\left[\begin{array}{c}
v_{1}^{-1} L_{1}\left(\psi_{1}\right) \\
\vdots \\
v_{N}^{-1} L_{N}\left(\psi_{N}\right)
\end{array}\right]+\left[\begin{array}{c}
f_{1}(t) \\
\vdots \\
f_{N}(t)
\end{array}\right]+\left[\begin{array}{c}
h_{1}\left(v_{1}^{-1} \psi_{1}\right) \\
\vdots \\
h_{N}\left(v_{N}^{-1} \psi_{N}\right)
\end{array}\right],
$$

where $A$ is now the simple matrix $J(1, V, 1)$, for $V=(-1,-2$, $\ldots,-2,-1)$. Let $P_{n}$ and $Q_{n}$ be the real and imaginary parts of $A_{n}$. By our assumptions, $P_{n}$ is selfadjoint and $Q_{n}$ is diagonal. For $z \in C^{N}$, we have

$$
\left(A_{n} z, A_{n} z=\left(P_{n} z, P_{n} z\right)+\left(Q_{n} z, Q_{n} z\right)+i\left(\left[P_{n}, Q_{n}\right] z, z\right)\right. \text {. }
$$

Let $C_{n}=\left[P_{n}, Q_{n}\right]=P_{n} Q_{n}-Q_{n} P_{n}$. Let

$$
X=\left(n\left(\frac{\gamma_{1}}{v_{1}}-\frac{\gamma_{2}}{v_{2}}\right), \ldots, n\left(\frac{\gamma_{n-1}}{v_{n-1}}-\frac{\gamma_{N}}{v_{N}}\right)\right)
$$

then, by direct calculation,

$$
C_{n}=J(X, 0,-X) \text {. }
$$

We now have

Lemma 3.7. Let $P_{n}, Q_{n}$ and $C_{n}$ be as above and suppose the assumptions of Corollary 2.2 hold. Then, for any $z \in C^{N}$, there are constants $K_{1}, K_{2}$ such that

(i) $0 \leq\left(P_{n} z, P_{n} z\right) \leq K_{1} n^{4}\|z\|^{2}$,

(ii) $\left|\left(C_{n} z, z\right)\right| \leq K_{2} n\|z\|^{2}$.

Proof. Since $P_{n}$ is a symmetric real matrix, we can estimate its norm by the square of its largest eigenvalue. This will be done by Gerschgorin's theorem (see [OP]). Let

$$
\varrho_{j}= \begin{cases}1 & j=1, N \\ 2 & \text { otherwise }\end{cases}
$$


and let

$$
\Lambda_{j}=\left\{z \in C \text { such that }\left|z-\left(P_{n}\right)_{j j}\right| \leq \varrho_{j}\right\} .
$$

Gerschgorin's theorem states that all the eigenvalues of $P_{n}$ lie in $\bigcup_{j=1}^{N} \Lambda_{j}$. Now

$$
\left|\left(P_{n}\right)_{j j}\right|^{2}=\left|\varrho_{j}-v_{j}^{-1}\left(\mu_{j}+n^{2}\right)\right|^{2} \leq \varrho_{j}^{2}+v_{j}^{-2}\left(\mu_{j}+n^{2}\right)^{2} .
$$

We can therefore estimate the modulus of the largest eigenvalue of $P_{n}$ by

$$
\left.\max _{j}\left(\mid P_{n}\right)_{j j} \mid+\varrho_{j}\right) \leq 2+\sqrt{2+\underline{v}^{-2}\left(\vec{\mu}+n^{2}\right)^{2}}
$$

it is therefore possible to find a constant $K_{1}$ such that (i) holds for all $n \in N$.

The proof of (ii) is also straightforward. Since $C_{n}^{T}=-C_{n}, i C_{n}$ is hermitian. We can therefore find a unitary matrix $U$ such that $U^{*}\left(i C_{n}\right) U$ is diagonal. Hence,

$$
\begin{aligned}
\left|\left(i C_{n}, z, z\right)\right| & =\left|\left(U^{*} i C_{n} U y, y\right)\right|, \\
& \leq \Lambda\|y\|=\Lambda\|x\|, \quad y=U^{*} x,
\end{aligned}
$$

where $\Lambda$ is the modulus of the largest eigenvalue of $C_{n}$. But by Gerschgorin's theorem, all the eigenvalues of $C_{n}$ have modulus smaller than

$$
\max _{j=1, \ldots, N-1}\left(\left|n\left(\tau_{j+1}+\tau_{j}\right)\right|, n\left|\tau_{1}\right|, n\left|\tau_{N}\right|\right)
$$

where $\tau_{j}=\left(\left(\gamma_{j+1} / v_{j+1}\right)-\left(\gamma_{j} / v_{j}\right)\right)$. We can therefore estimate (3.14) by

$$
\frac{n}{N} \frac{b \bar{v}+c \bar{\gamma}}{\underline{v}^{2}}
$$

This proves (ii).

Using Lemma 3.7 and (3.11) we can now prove

Proposition 3.8. Let $A_{n}$ be defined by (3.11) and suppose the assumptions of Corollary 2.2 hold. Then there are constants $\Lambda_{0}, \Lambda_{1}$ such that

$$
\Lambda_{0} n \leq\left\|A_{n}\right\| \leq \Lambda_{1} n^{2}
$$

Proof. Let $z \in C^{N}$. By Lemma 3.7 and (3.11),

$$
\left(A_{n} z, A_{n} z\right) \leq\left(K_{1} n^{4}+\underline{v}^{-2} \bar{\gamma}^{2} n^{2}+K_{2} n\right)\|z\|^{2}
$$

and we can therefore estimate $\left\|A_{n}\right\|$ by $\Lambda_{1} n^{2}$ for some $\Lambda_{1}$. Similarly, using (3.16)

$$
\left(A_{n} z, A_{n} z\right) \geq\left(n^{2} \bar{v}^{-2} \underline{\gamma}^{2}-\frac{n}{N} \frac{b \bar{v}+c \bar{\gamma}}{\underline{v}^{2}}\right)\|z\|^{2} .
$$


By our assumptions, the right hand side of (3.17) is positive and we can therefore estimate $\left\|A_{n}\right\|$ from below by $\Lambda_{0} n$.

Since this proposition implies $(2.1)$, with $p_{0}=1, p_{1}=2$, the corollary is proved.

\section{Approximation theory}

The aim of this section is not so much to give yet another theory for approximations as to show how the method of the existence proof can be used to check the validity of the approximation. That is, we do not wish to develop a new and consistent approximation theory, for which several books and papers can be found, see for example D. Braess [Br] or E. W. Cheney [Ch]. Rather, we wish to address the following problem: Given an approximate solution to the discrete system, or to the continuous system, what is the "convergence" of the approximate solution to the exact solution. More precisely, by how much does the approximate solution fail to be an exact solution. The basic idea is to use the operator $B$ which was defined in the proof of Theorem 2.1 to check by how much the approximate solution fails to be a true solution of the discrete equation. Since the operator $B$ is known, the only tool we need are Taylor series. Plugging these into (1.5), one obtains the Theorem 2.3.

Throughout this section, we will assume the correspondence (1.3). Furthermore, we will assume that $f, \mu, v$ and $\gamma$ are $C^{(2)}$ in $x$ and that for any $x \in[0,1], f(t, x)$ is $H^{3}\left(S^{1}\right)$ in $t$. Under the assumptions of Corollary 2.2, let $\phi(t)$ be the solution of the discrete equation. Let

$$
u_{0}(t, x)=-\sum_{n} \frac{f^{(n)}(x)}{\mu(x)-n^{2}+i \gamma(x) n} e^{i n t} .
$$

Denote

$$
\xi_{k}=\frac{1}{N} u_{0}\left(\frac{k}{N}\right), \quad k=1, \ldots, N .
$$

By the definition of $u_{0}$, we immediately have

$$
\xi_{k}=\gamma_{k} \dot{\xi}_{k}+\mu_{k} \xi_{k}+f_{k}=0 .
$$

Let

$$
\xi=\left(\begin{array}{c}
\xi_{1} \\
\vdots \\
\xi_{N}
\end{array}\right)
$$


We write

$$
B \xi=f(t)+h(t, \xi, \dot{\xi})+R
$$

where for $k=2, \ldots, N-1$,

$$
R_{k}=v_{k+1} \xi_{k+1}+2 v_{k} \xi_{k}+v_{k-1} \xi_{k-1}-h_{k}\left(t, \xi_{k}, \dot{\xi}_{k}\right)
$$

and where

$$
\begin{aligned}
& R_{1}=-v_{2} \xi_{2}+v_{1} \xi_{1}-h_{1}\left(t, \xi_{1}^{\prime} \dot{\xi}_{1}\right), \\
& R_{N}=v_{N} \xi_{N}-v_{N-1} \xi_{N-1}-h_{N}\left(t_{1}, \xi_{N}, \dot{\xi}_{N}\right) .
\end{aligned}
$$

We now estimate the residual $R$. For $k=2, \ldots, N$, expanding $v((k+1) / N) u(t,((k+1) / N))$ in Taylor expansion around $(k / N)$, we have

$$
\begin{aligned}
v_{k \pm 1} \xi_{k \pm 1}= & \frac{1}{N} v\left(\frac{k}{N}\right) u\left(t, \frac{k}{N}\right) \pm \frac{1}{N^{2}}\left(v\left(\frac{k}{N}\right) u\left(t, \frac{k}{N}\right)\right)_{x} \\
& +\left.\frac{1}{2 N^{3}}(v(x) u(t, x))_{x x}\right|_{x=\delta_{k \pm 1}},
\end{aligned}
$$

where $\left|\delta_{k \pm 1}-(k / N)\right| \leq 1 / N$. We therefore have

$$
\begin{aligned}
& v_{k+1} \xi_{k+1}-2 v_{k} \xi_{k}+v_{k-1} \xi_{k-1} \\
& \quad=\frac{1}{2 N^{3}}\left(\left(v\left(\delta_{k \pm 1}\right) u_{0}\left(t, \delta_{k+1}\right)\right)_{x x}+\left(v\left(\delta_{k-1}\right) u_{0}\left(t, \delta_{k-1}\right)\right)_{x x}\right) .
\end{aligned}
$$

Similarly, when $k=1$ or $N$, we have

$$
\begin{aligned}
v_{2} \xi_{2}-v_{1} \xi_{1}= & \frac{1}{N^{2}}\left(\left(v\left(\delta_{1}\right) u_{0}\left(t, \delta_{1}\right)\right)_{x}\right), \\
& -v_{N} \xi_{N}+v_{N-1} \xi_{N-1}=\frac{1}{N^{2}}\left(\left(v\left(\delta_{N}\right) u_{0}\left(t, \delta_{N}\right)\right)_{x}\right),
\end{aligned}
$$

where $\left|\delta_{1}-(1 / N)\right| \leqslant 1 / N,\left|\delta_{N}-(1 / N)\right| \leq 1 / N$. We now only need to estimate the nonlinearity. By the Sobolev embedding theorems, we have

$$
\sup _{t \in S^{1}, x \in[0,1]}\left|u_{0}(t, x), \frac{\partial}{\partial t} u_{0}(t, x)\right| \leq C \max _{n} \sup _{x \in[0,1]} \frac{\|f(t, x)\|_{H^{3}\left(S^{1}\right)}}{\left|\mu(x)-n^{2}+i \gamma(x) n\right|},
$$

where $C$ is the Sobolev constant and where $f(t, x)=\sum_{n} f^{(n)}(x) e^{\text {int }}$. We will denote the right hand side of (4.7) by $K(f, \mu, \gamma)$. By our assumptions on $h$, each $h_{k}$ is quadratic in $\phi$ and $\dot{\phi}$. Hence, there exists a constant $\chi(h)$ such that

$$
\|h(t, \xi, \dot{\xi})\|_{1} \leq \frac{\chi(h)}{N^{3 / 2}} K(f, \mu, \gamma)
$$


By (4.5), (4.6) and (4.8), using the definition of the $\mathscr{H}_{l}$ norm for $l=1$, we obtain

$$
\begin{aligned}
\|R\|_{1} \leq & \frac{1}{N^{3 / 2}}\left(\chi(h) K(f, v, \gamma)+\frac{1}{N^{1 / 2}}\right. \\
& \left.\times \max \left(\sup _{t \in S^{1}}\left(\left\|u_{0}(t, x)\right\|_{C^{3}},\left\|\frac{\partial}{\partial t} u_{0}(t, x)\right\|_{C^{3}}\right)\right)\right)^{2} .
\end{aligned}
$$

Since

$$
\phi-\xi=B^{-1}(h(t, \phi, \dot{\phi})-h(t, \xi, \dot{\xi})+R),
$$

we have

$$
\|\phi-\xi\|_{1} \leq\left\|B^{-1}\right\|\left(\|h(t, \phi, \dot{\phi})-h(t, \xi, \dot{\xi})\|_{1}+\|R\|_{1}\right) .
$$

By (3.4) and since by Proposition 2.6, $\|h(t, \phi, \phi)-h(t, \xi, \dot{\xi})\|_{1} \leq \delta\|\phi-\xi\|_{1}$ for some $\delta<1$, we finally have

$$
\|\phi-\xi\|_{1} \leq \frac{1}{(1-\delta) \Lambda_{0}}\|R\|_{1}
$$

and by the definition of \|\|$_{l}$,

$$
\sup _{t \in S^{1}}\left|\phi_{k}(t)-\xi_{k}(t)\right| \leq C\|\phi-\xi\|_{1} \text {. }
$$

This concludes the proof of Theorem 2.3. Note that the constant in (4.9) is of order $\left(\sup _{x \in[0,1]}\|f\|_{H^{3}\left(S^{1}\right)}\right)^{2}$.

Remark 4.1. When $h=0$, we have in fact a sharper estimate, namely

$$
\sup _{t \in S^{1}}\left|\phi_{k}(t)-\xi_{k}(t)\right| \leq \frac{K_{5}}{N^{5 / 2}} \text {. }
$$

In the case where the linear terms of $h$ are of order one, our argument does not apply any more. In this case, for the corresponding continuous equation, one does not have a $1 / N$ in front of the function $h$, so the theorems of [4] do not apply any more.

Remark 4.2. The $\phi_{k}$ 's are themselves of order $1 / N$. So if we scale them to order one, we see that for big $N$, the $u(t, k / N)$ are still within $O\left(N^{-1 / 2}\right)$ of the scaled $\phi_{k}$ 's.

\section{References}

[Br] D. Braess, Nonlinear Approximation Theory, Springer Series in Computational Maths., Vol. 7, Springer Verlag, Berlin 1986.

[B] G. Bowtell, personal communication.

[Ch] E. W. Cheney, Introduction to Approximation Theory, McGraw-Hill, New York 1966. 
[Co] V. Coppel, Dichotomies in Stability Theory, LNM 629, Springer Verlag, Berlin 1978.

[EK] N. Kopell and G. B. Ermentrout, Symmetry and phase locking in chains of weakly coupled oscillators, Comm. in Pure and Appl. Math. 39, 263-360 (1986).

[H] J. Hale, Asymptotic behaviour of dissipative systems, AMS mathematical surveys and monographs 25, 1988.

[HR] J. Hale and G. Raugel, personal communication.

[Le] M. Levi, Beating Modes in the Josephson Junction, Conley's memorial issue, Erg. Th. and Dyn. Syst. 1988, Vol. 8 .

[LL] S. Laederich and M. Levi, Qualitative Dynamics of Planar Chains, Preprint, 1989.

[La] S. Laederich, Boundary value problems for partial differential equations with exponential dichotomies, preprint.

[OP] J. M. Ortega and W. G. Poole, Jr., Introduction to Numerical Methods for Differential Equations, Putnam, 1981.

[P] R. S. Palais, Foundations of Global Non Linear Analysis, Benjamin, New York 1968.

[Pa] A. Pazy, Semigroups of Linear Operators and Applications to Partial Differential Equations, Springer, New York 1983.

\begin{abstract}
In this paper we will be interested in the behaviour of long chains of coupled gravitational pendula. We will prove existence and uniqueness of periodic solutions for such chains under periodic forcing and will prove that under some smoothness assumptions the chain behaves as an uncoupled one. We will also analyse a more general class of differential difference equations and prove existence and unicity results for periodic solutions.
\end{abstract}

(Received: April 19, 1990) 